\title{
STRATIGRAPHIC SECTIONS
}

\section{OF THE PHOSPHORIA}

\section{FORMATION IN WYOMING}

1952

This report concerns work done on behalf of the U. S. Atomic Energy Commission and is published with the permission of the Commission. 
UNITED STATES DEPARTMENT OF THE INTERIOR

Douglas McKay, Secretary

GEOLOGICAL SURVEY

W. E. Wrather, Director

GEOLOGICAL SURVEY CIRCULAR 325

\section{STRATIGRAPHIC SECTIONS OF THE PHOSPHORIA FORMATION IN WYOMING}

\section{2}

By R. P. Sheldon, E. R. Cressman, L. D. Carswell, and R. A. Smart

This report concerns work done on

behalf of the U. S. Atomic Energy

Commission and is published with

the permission of the Commiseion. 


\section{STRATIGRAPHIC SECTIONS OF THE PHOSPHORIA FORMATION IN WYOMING}

\section{2}

By R. P. Sheldon, E. R. Cressman, L. D. Carswell, and R. A. Smart

\section{CONTENTS}

Lot no. Page

Lot no. Page

Introduction. . . . . . . . . . . . .

Acknowledgments.................

Stratigraphy of the Phosphoria

formation in western Wyoming.....

Stratigraphic sections.............

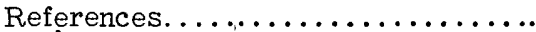

Tables of stratigraphic

sections

Middle Piney Lakes..........

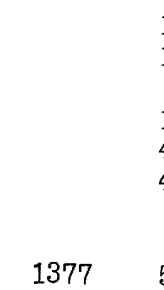

1

1

4

4

5
Tables of stratigraphic sections-Continued

Deadline Ridge.............. $1380 \quad 8$

Fontenelle Creek.............................. 1379

Basin Creek................ 1381

West Branch of

Hams Fork Creek. .......... $1383 \quad 19$

Wheat Creek. ................ $1378 \quad 20$

South Mountain pit and mine...... $1382 \quad 23$

\section{ILLUSTRATIONS}

Figure 1. Outcrops of the Phosphoria formation in Wyoming and localities sampled..................

2. Generalized section of the Phosphoria formation at Coal Canyon, Wyoming [lot no. 1201]......

\section{INTRODUCTION}

The U. S. Geological Survey has measured and sampled the Phosphoria formation of Permian age at many localities in Wyoming and adjacent states. The data will not be fully synthesized for many years, but segments of the data, accompanied by little or no interpretation, are published as preliminary reportis as they are assembled. This report, which contains abstracts of the sections measured in western Wyoming (fig. 1) during 1952, is the fourth Wyoming report of this series. The field and laboratory procedures adopted in these investigations are described in a previous report (McKelvey and others, 1953a).

Many people have taken part in this investigation. T. M. Cheney participated in the description of strata and the collection of samples referred to in this report and T. K. Rigby assisted in the collection of samples. The laboratory preparation of samples for chemical analysis was done in Denver, Colo., under the direction of L. F. Rader. The chemical analyses were made for the Survey by the U. S. Bureau of Mines at the Northwest Electrodevelopment Laboratory, Albany, Oreg., under the direction of S. M. Shelton and M. L. Wright.

K. S. Bergman compiled most of the data and Anita. Wise organized the tabular data.

\section{ACKNOWLEDGMENTS}

Special thanks are due J. D. Love, W. W. Rubey, and J. Steele Williams, who contributed much in the way of advice and suggestions in planning and organization of the field program: The cost of both the field and laboratory investigations has been borne partly by the Division of Raw Materials of the Atomic Energy Commission. It is a pleasure to acknowledge the fine cooperation extended to the field parties by the local residents, property owners, and phosphate companies, who furnished information and services and gave access to property.

\section{STRATIGRAPHY OF THE PHOSPHORIA FORMATION IN WESTERN WYOMING}

In northwestern Wyoming the Phosphoria formation is about 200 feet thick and has been divided into five members. These members have been tentatively correlated with the five members in Montana that are designated the $A, B, C, D$, and $E$, from oldest to youngest (Klepper and others in McKelvey, 1949). Member A, overlies the Tensleep sandstone of Pennsylvanian age and consists of cherty carbonate and detrital rocks; it may be equivalent to the upper part of the Wells formation in Idaho. Members $B$, $C$, and $D$, composed respectively of phosphatic, cherty, 


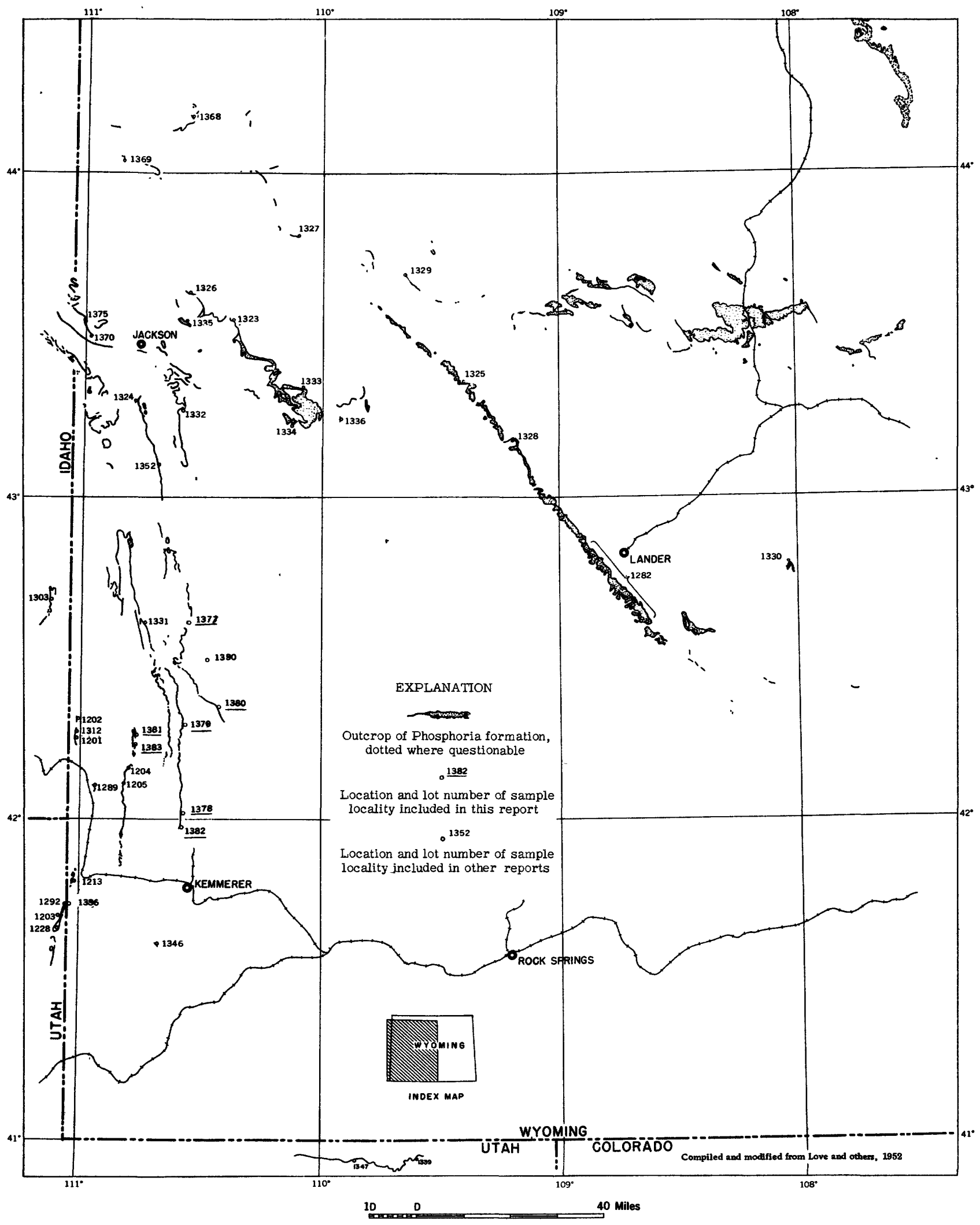

Figure 1. Outcrops of the Phosphoria formation in Wyoming and localities sampled. 


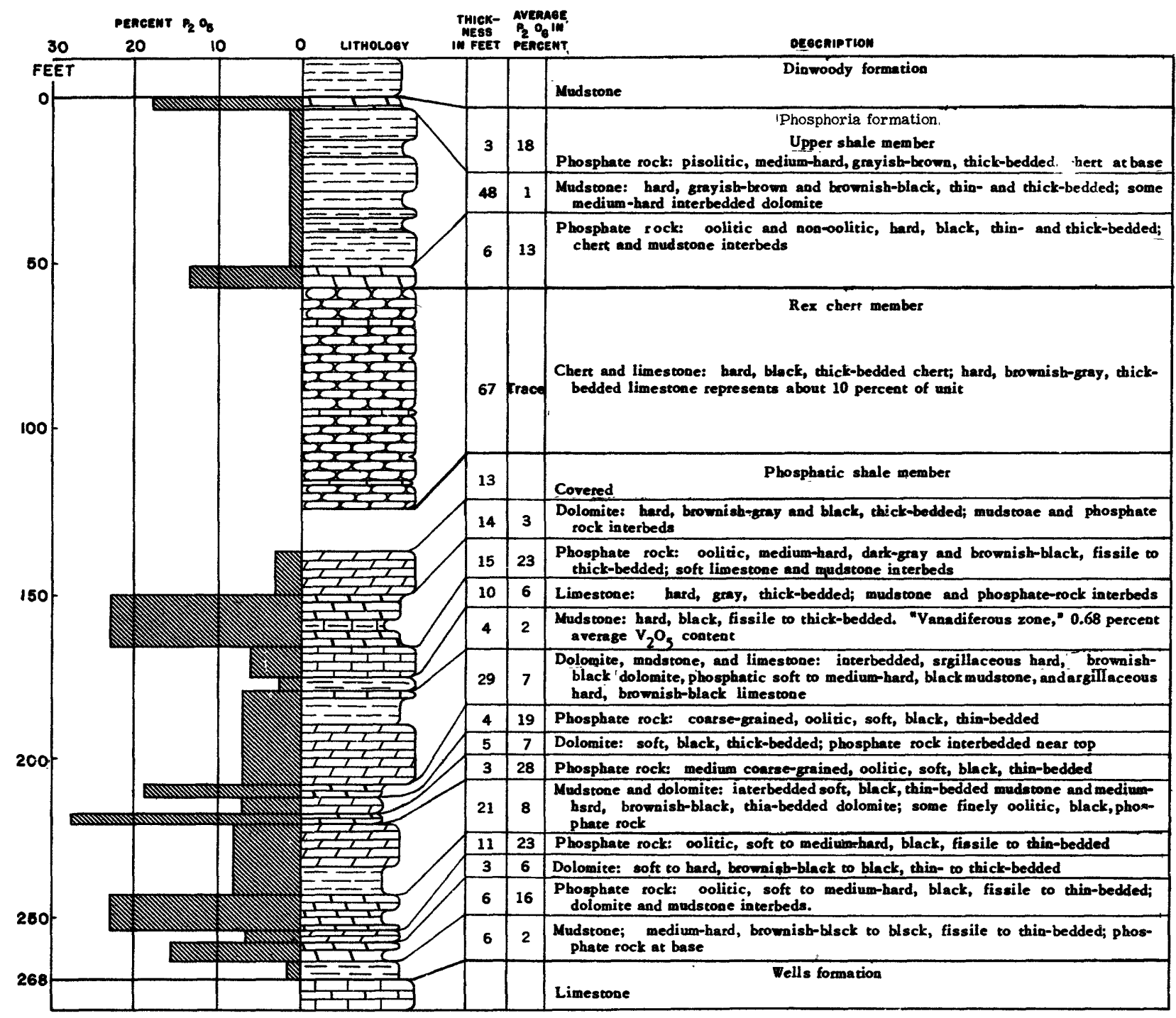

Figure 2. Generalized section of the Phosphoria formation at Coal Canyon, Wyoming (lot no. 1201). 
and phosphatic rocks, are equivalent to the phosphatic shale member, the Rex chert member, and the upper shale member of the Phosphoria formation in Idaho and southwestern Wyoming (Sheldon in Swanson and others, 1953). Member $E$, not yet $\overline{r e c}$ ognized in southeastern Idaho, consists of chert, sandstone, and carbonate rock and is overlain by the Dinwoody formation of Triassic age.

In southwestern Wyoming the Phosphoria formation consists of 3 members: a lower phosphatic shale member, 95-145 feet in thickness; the medial Rex chert member, cherty limestone, 65-145 feet in thickness; and the upper shale member, 15-60 feet in thickness (fig. 2). It overlies the Wells formation of Pennsylvanian age and underlies the Dinwoody formation of Triassic age. Although the Wells formation consists largely of quartzose sandstone, calcareous in part; the upper 25 feet or more is dark-gray limestone and is equivalent to the upper member of the Wells formation in Idaho and may be the correlative of the lowermost member (A member) of the Phosphoria formation in northwestern Wyoming and Montana and, according to McKelvey, the lower limestone member of the Park City formation in Utah. The Dinwoody formation consists of limestone, calcareous siltstone, and sandstone.

The correlation of individual beds of the phosphatic shale members with those in adjacent parts of Idaho, Montana, and Utah will be discussed in greater detail in a later publication. The phosphatic shale member in southwestern Wyoming and its correlative, the $\mathrm{B}$ member, in northwestern Wyoming contain most of the phosphate-bearing layers. The upper shale member and its correlative, the $D$ member, contain only thin layers of phosphatic chert and phosphatic layers of local significance.

\section{STRATIGRAPHIC SECTIONS}

Analytical data and abstracts of stratigraphic sections measured at seven localities follow. Their locations, as well as the locations of those reported previously (McKelvey and others, 1953b, Sheldon and others, 1953, Cheney anci others, 1953) and of others to be reported later, are shown in figure 1.

\section{REFERENCES}

Cheney, T. M., Sheldon, R. P., Waring, R. G., and Warner, M. A., 1953, Stratigraphic sections of the Phosphoria formation in Wyoming, 1951: U. S. Geol. Survey Circ. 324.

Love, J. D., Weitz, J. L., and Hose, R. K., 1952, Geologic map of Wyoming: U. S. Geol. Survey.

McKelvey, V. E., 1949, Geological studies of the western phosphate field: Am. Inst. Min. Met. Eng. Mining Trans., v. 184, p. 270-279.

McKelvey, V. E., Davidson, D. F. , O'Malley, F. W. and Smith, L. E., 1953a, Stratigraphic sections of the Phosphoria formation in Idaho, part 1: U. S. Geol. Survey Circ. 208.

McKelvey, V. E., Smith, L. E., Hoppin, R. A., and Armstrong, F. C., 1953b, Stratigraphic sections of the Phosphoria formation in Wyoming, 1947-48: U. S. Geol. Survey Circ. 210.

Sheldon, R. P., Waring, R. G., Warner, M. A., and Smart, R. A., 1953, Stratigraphic sections of the Phosphoria formation in Wyoming, 1949-50: U. S. Geol. Survey Circ. 307.

Swanson, R. W., McKelvey, V. E., and Sheldon, R. P., 1953, Progress report of investigations of northwest phosphate deposits: U. S. Geol. Survey Circ. 297. 


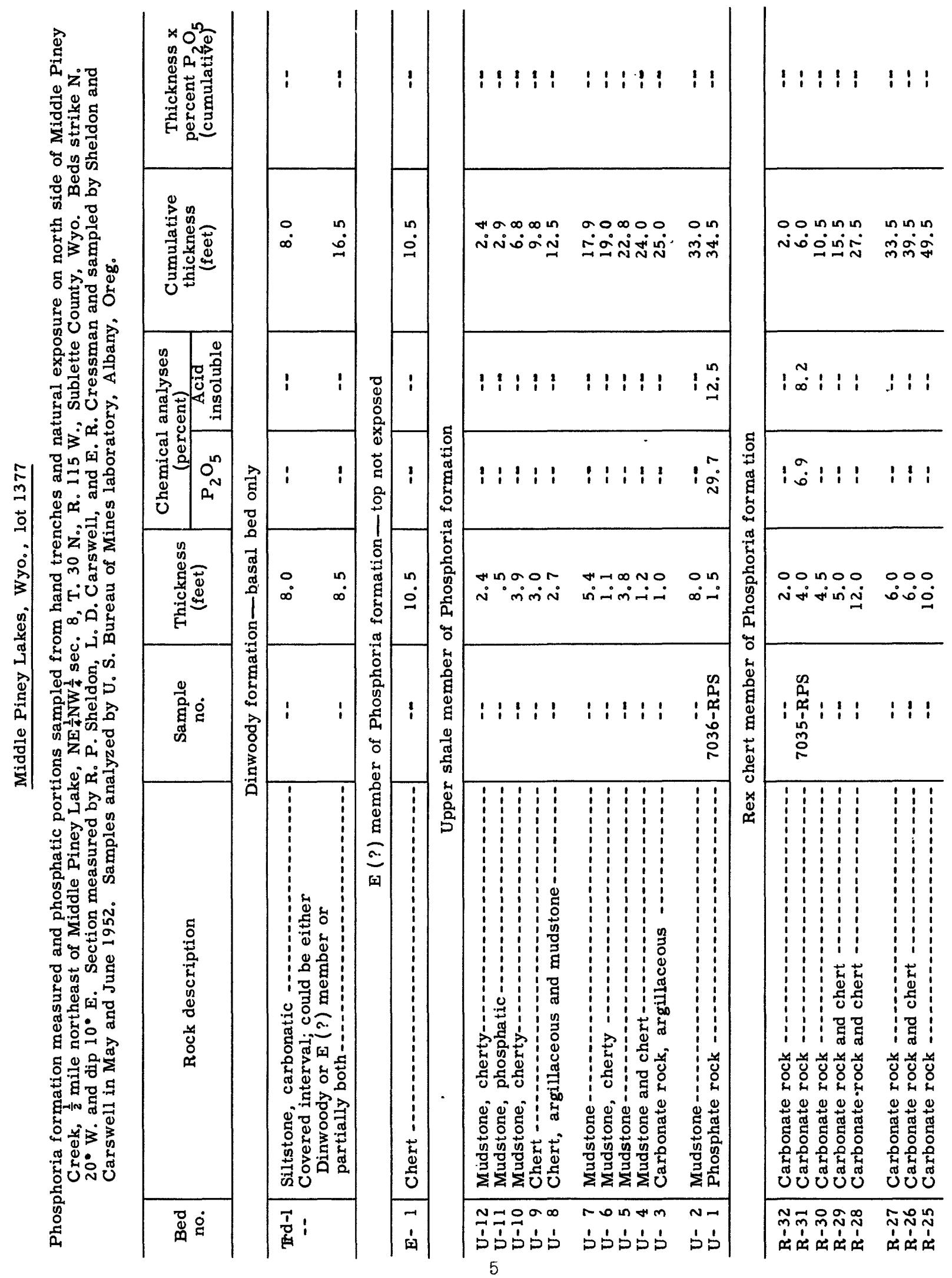




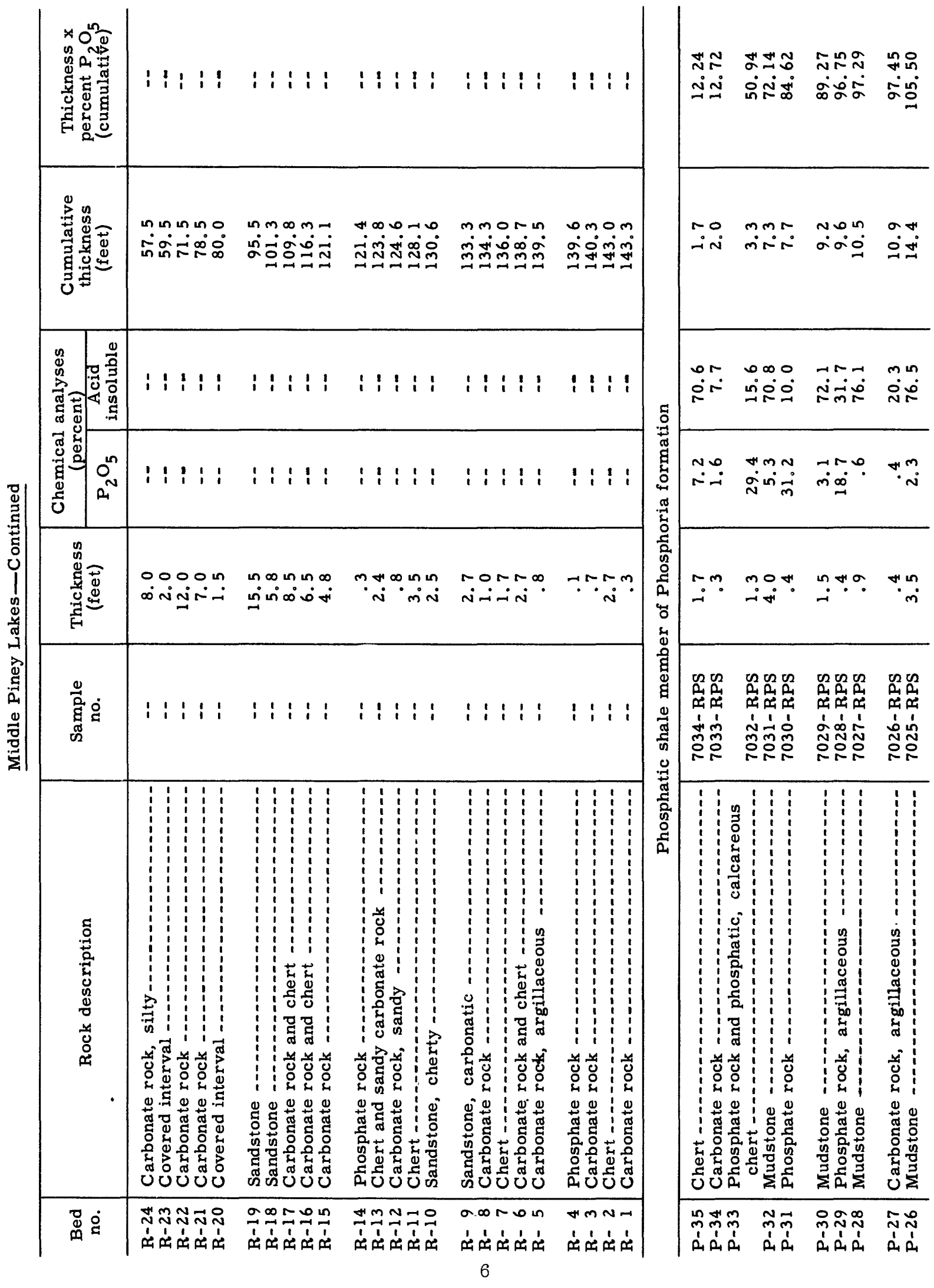


윰ำ

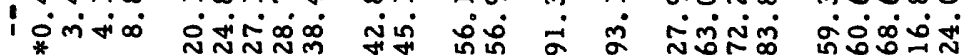

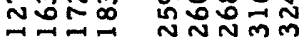

11:1: $1: 1: 1$ :

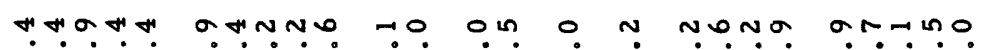

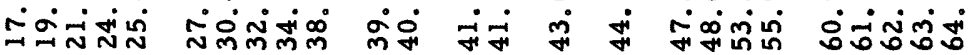

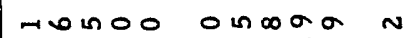

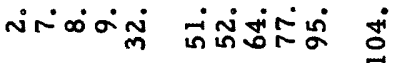

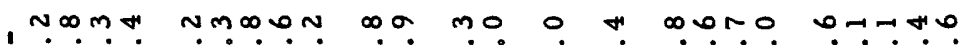

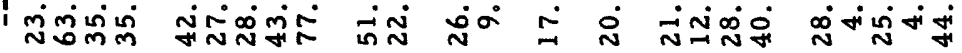

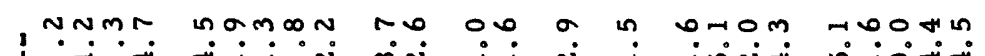

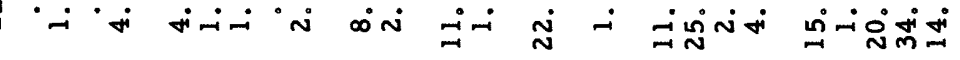

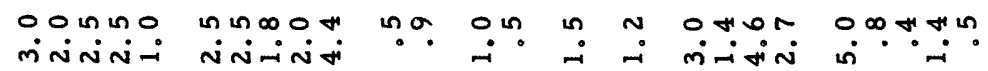

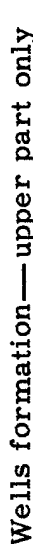

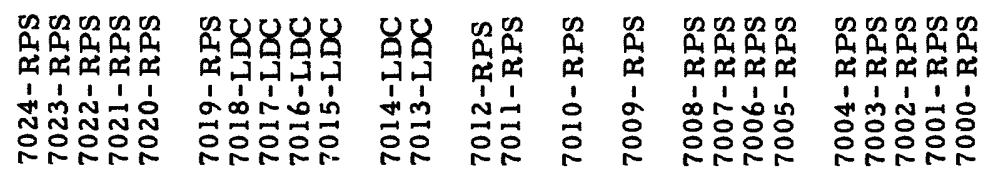

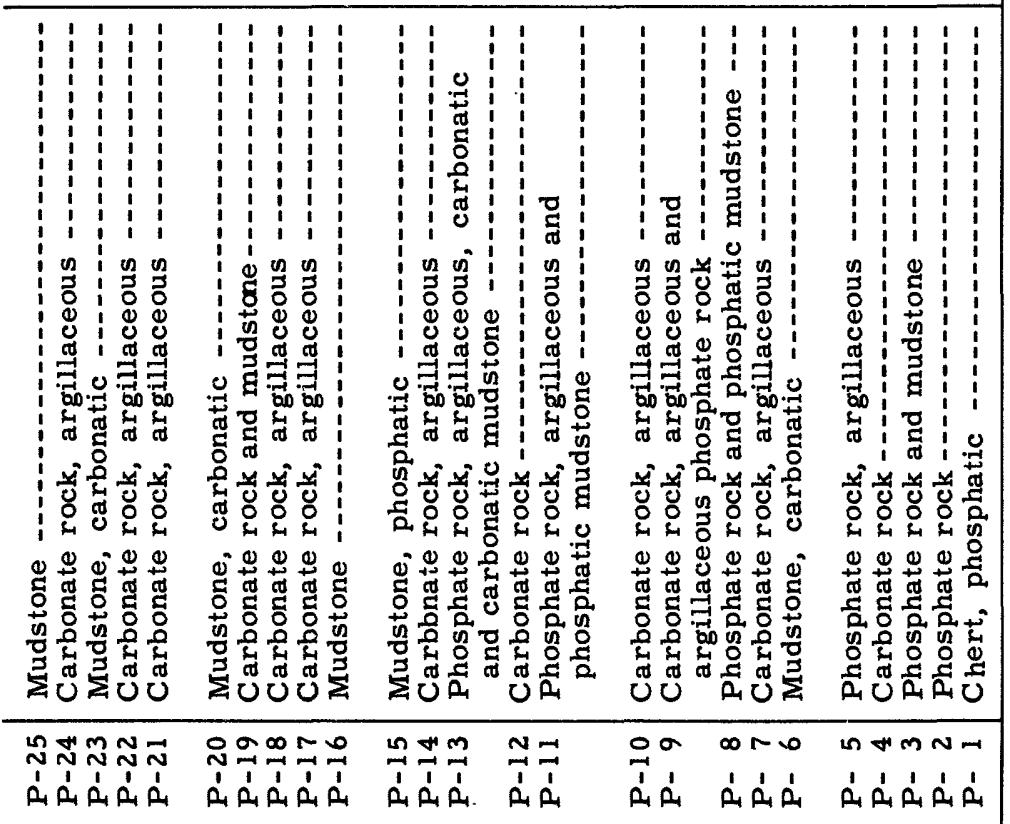

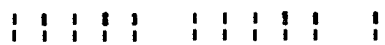

Hnomo onmmo m

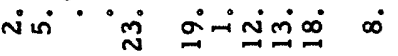

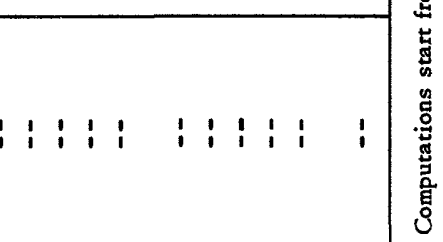

:

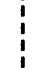

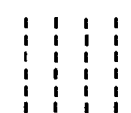

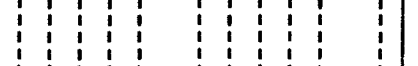

(1)

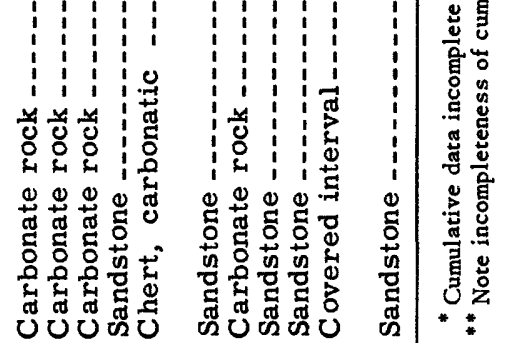

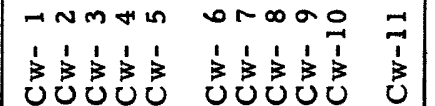




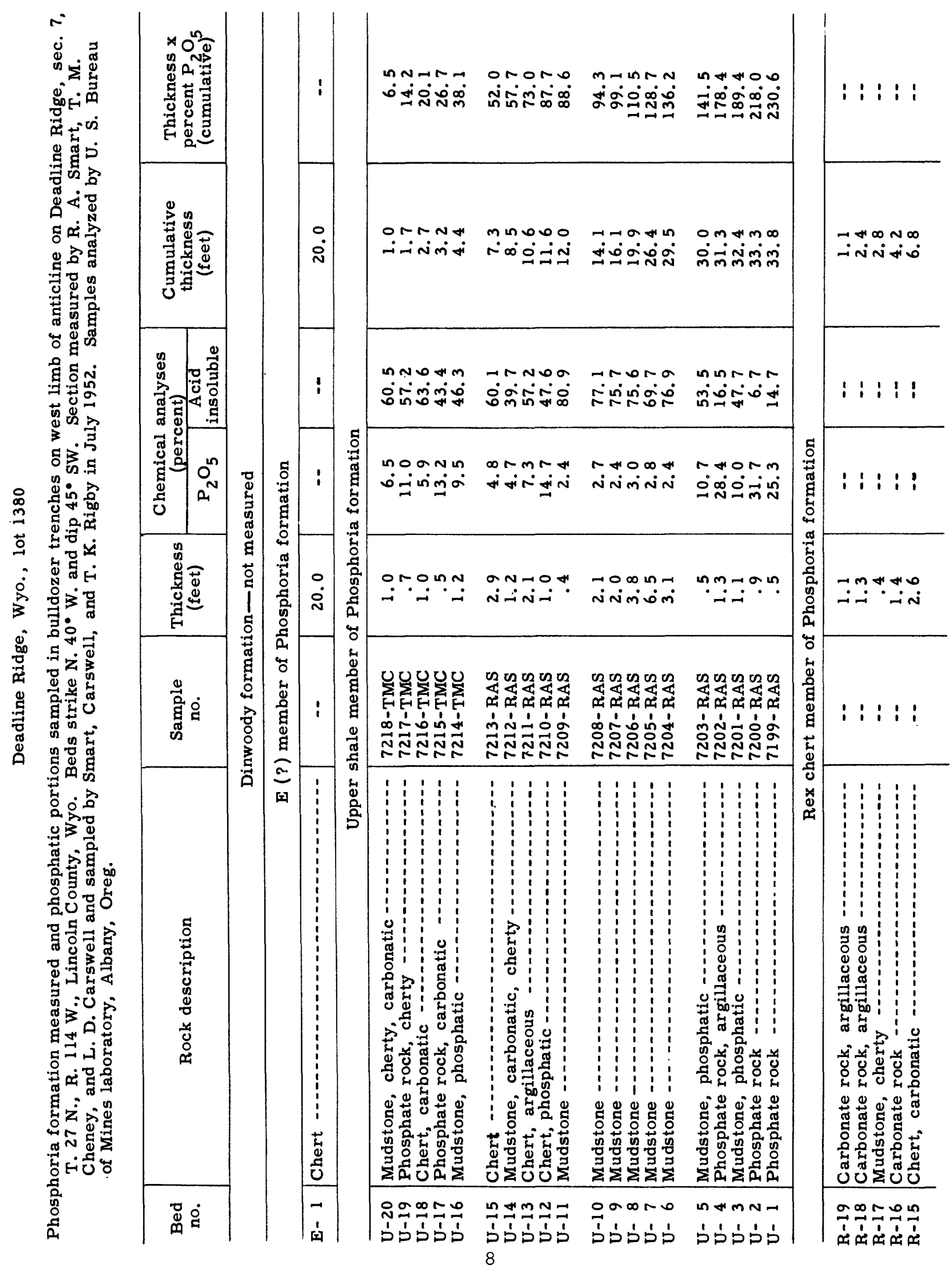




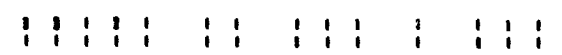

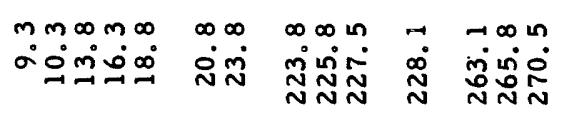

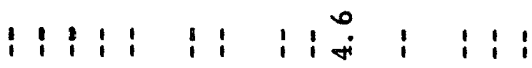

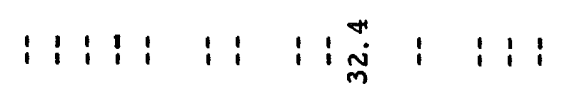

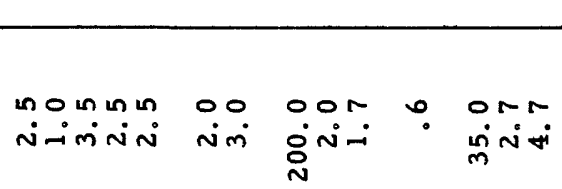

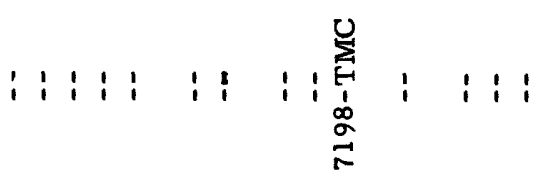

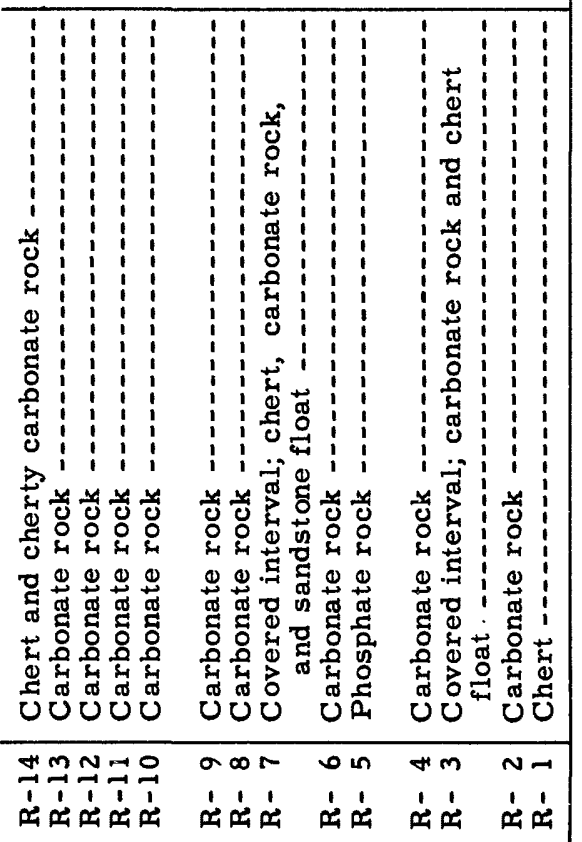

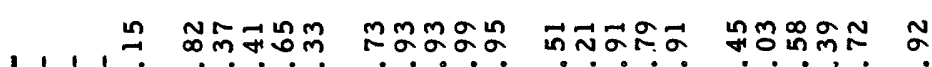

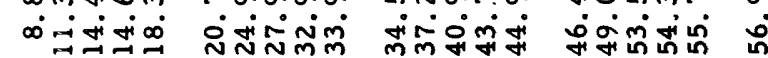

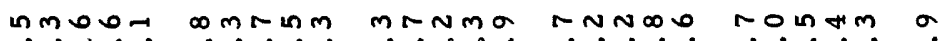

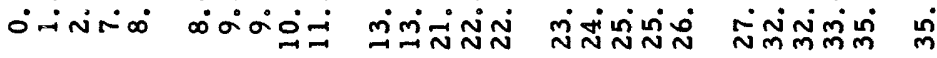

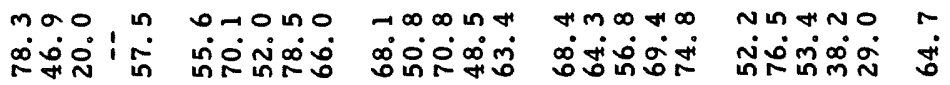
:

onn m 帘

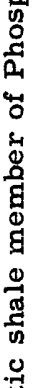

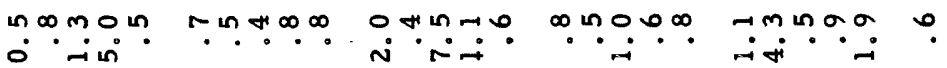

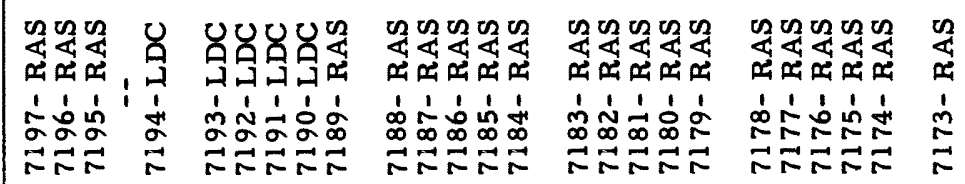

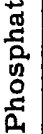

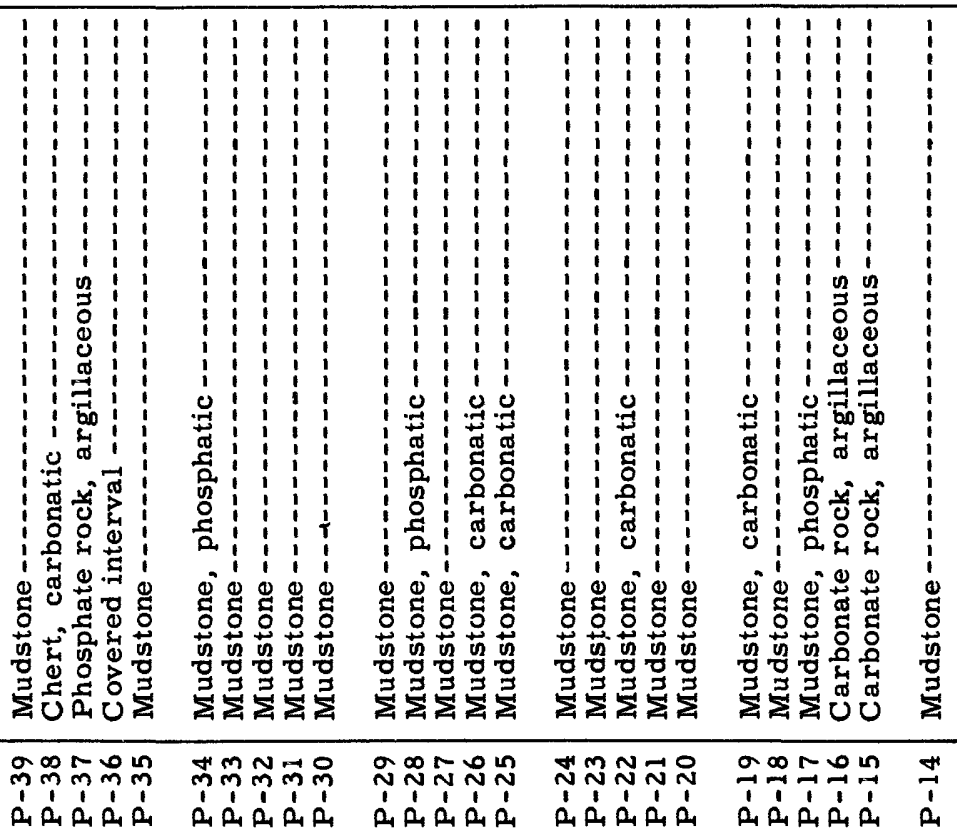




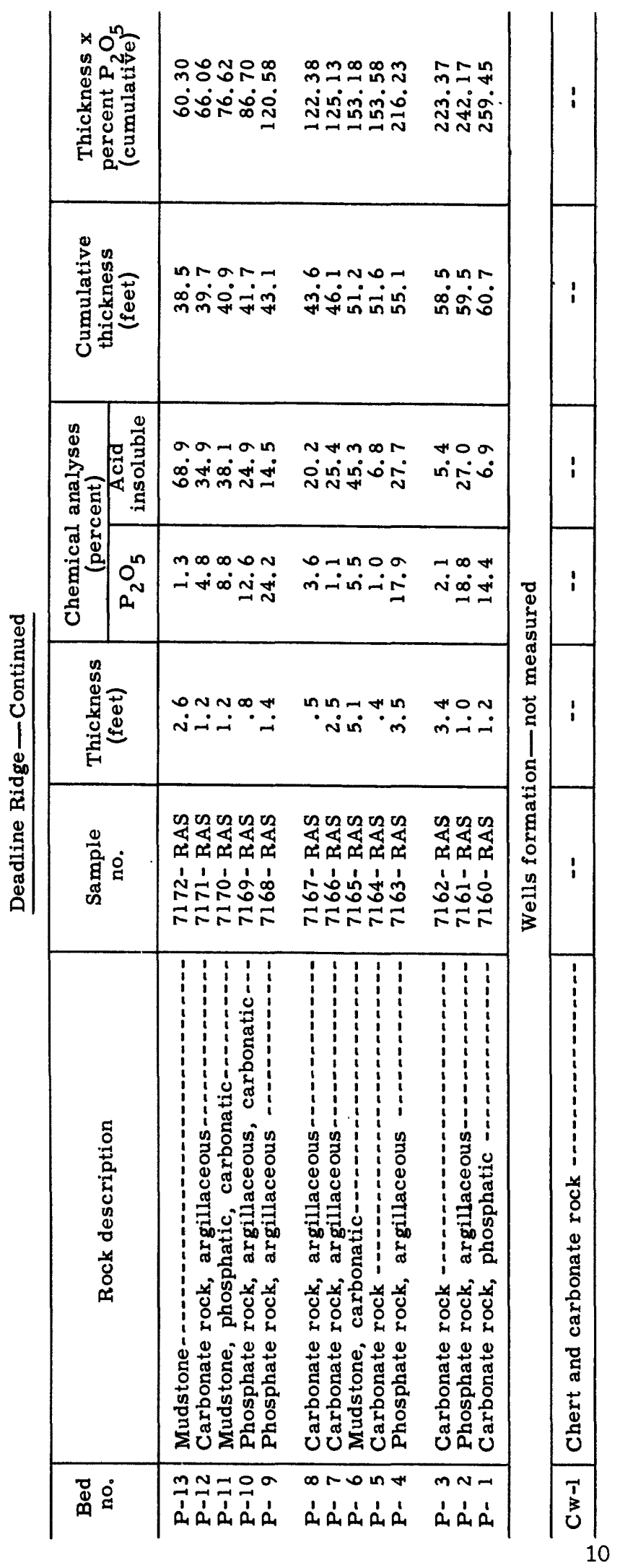




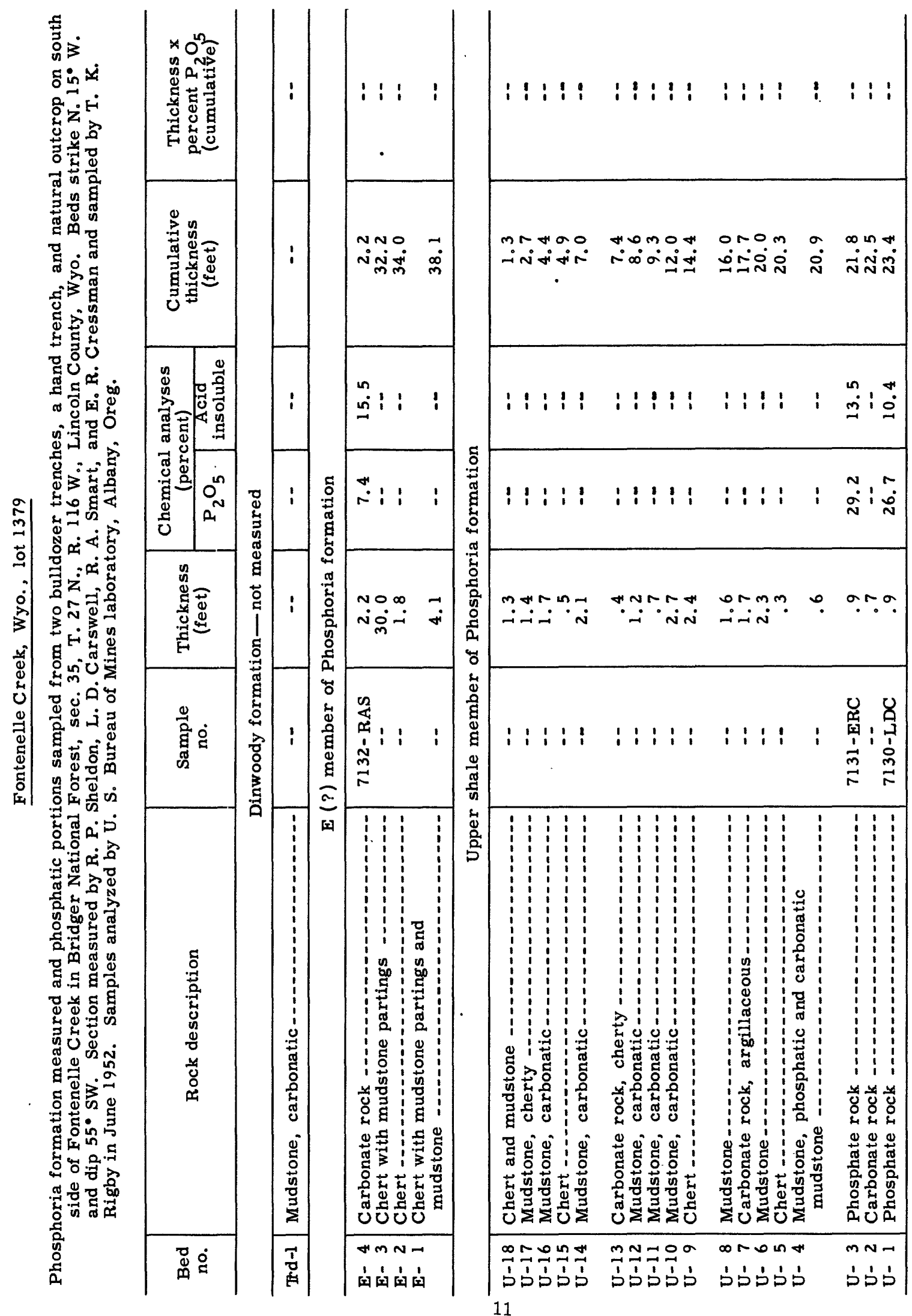




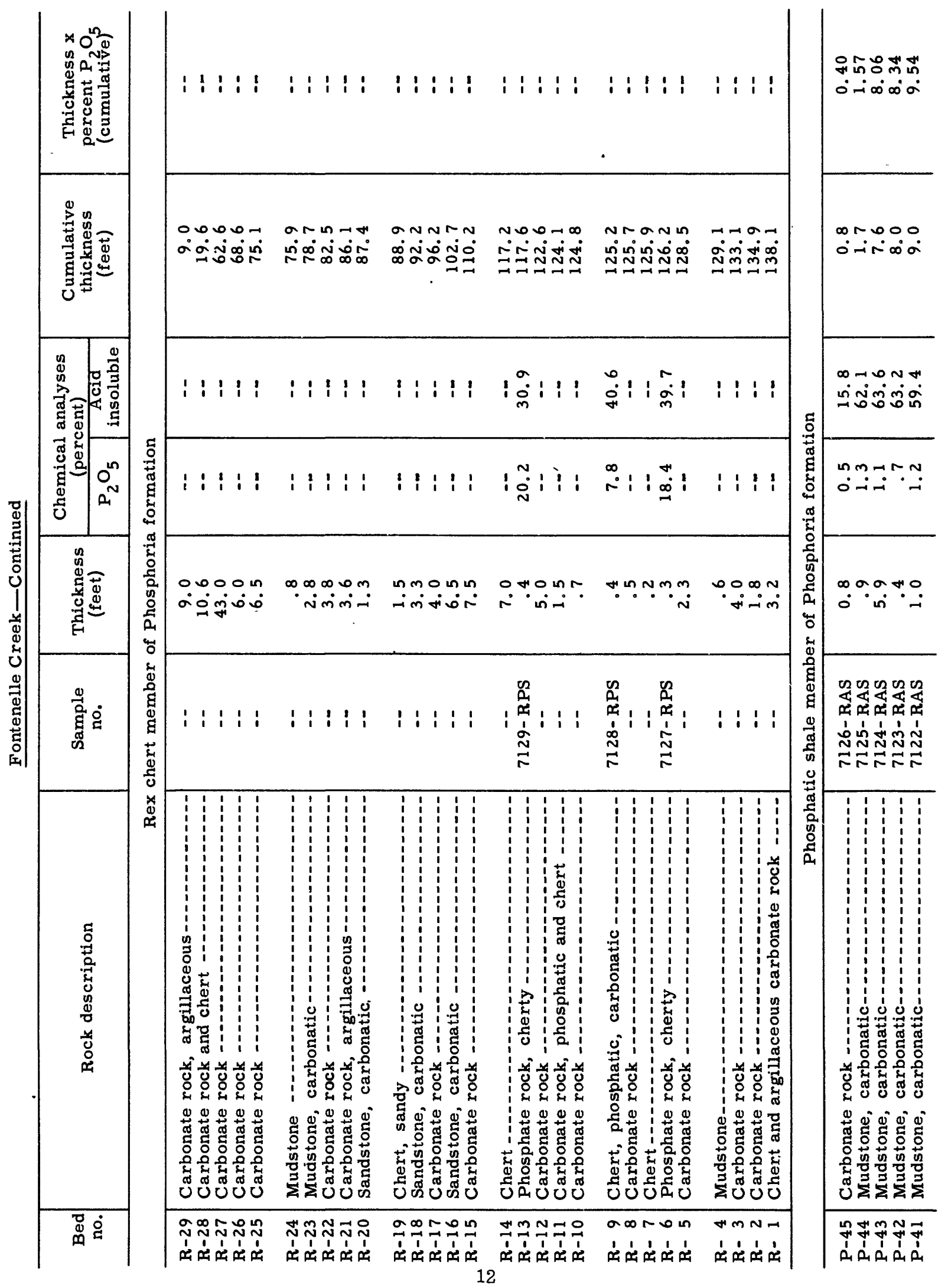




\begin{tabular}{|c|c|c|c|c|c|c|c|}
\hline 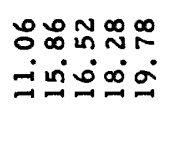 & 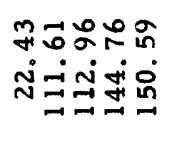 & 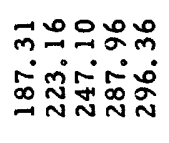 & 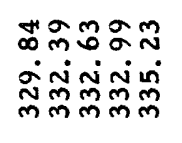 & 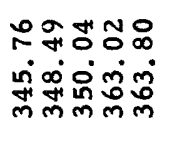 & 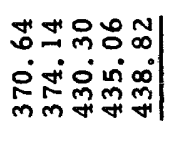 & 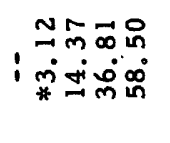 & 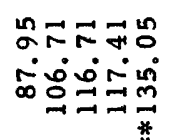 \\
\hline
\end{tabular}

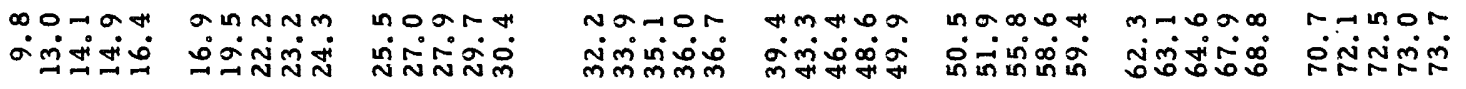

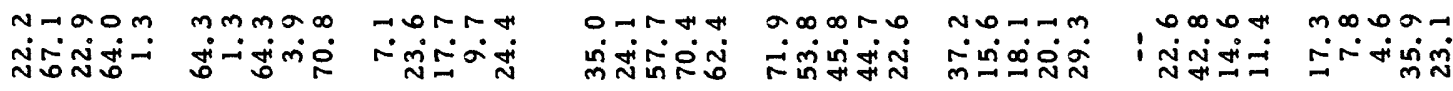

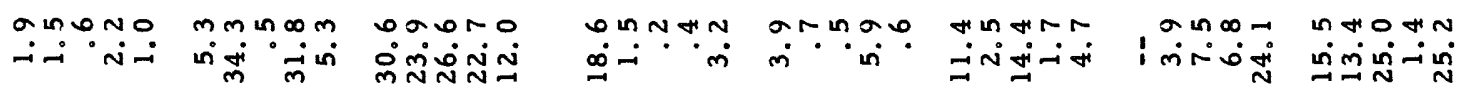

omĩ.

\begin{tabular}{|c|c|c|c|c|c|c|c|}
\hline 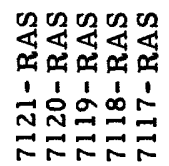 & 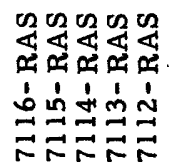 & 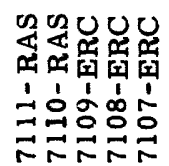 & 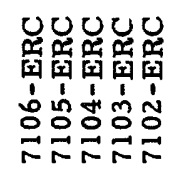 & 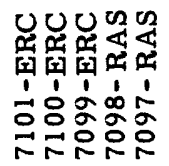 & 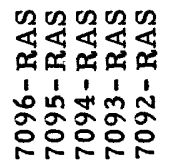 & 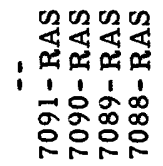 & 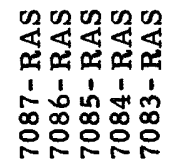 \\
\hline
\end{tabular}

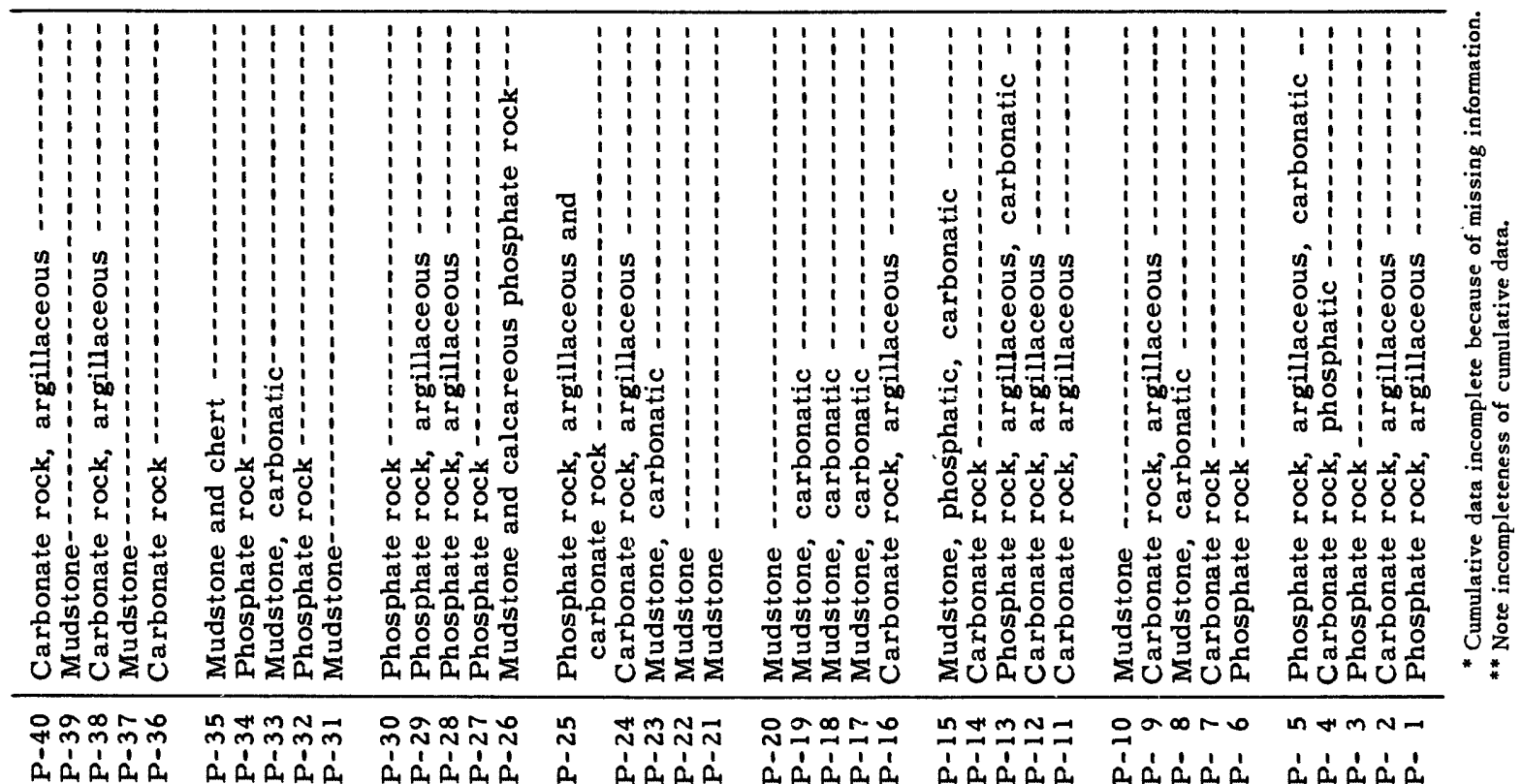




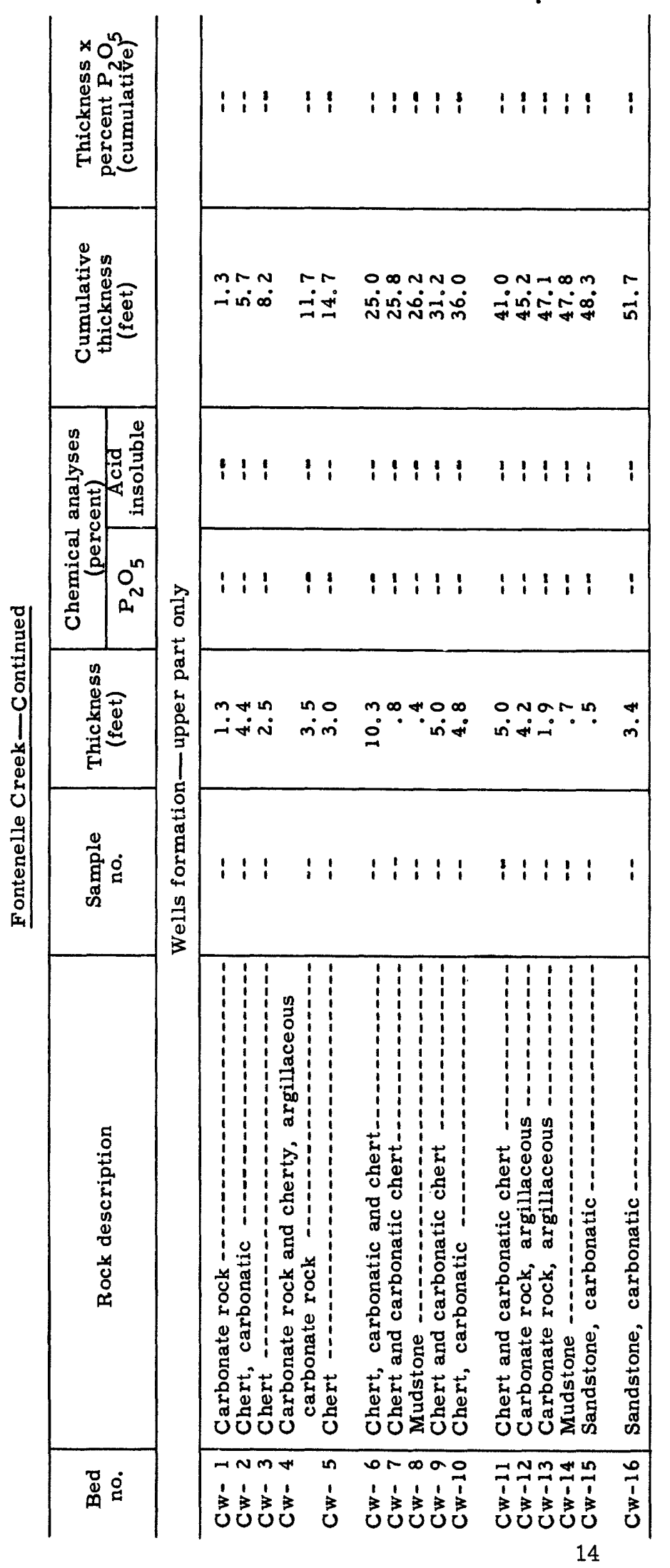




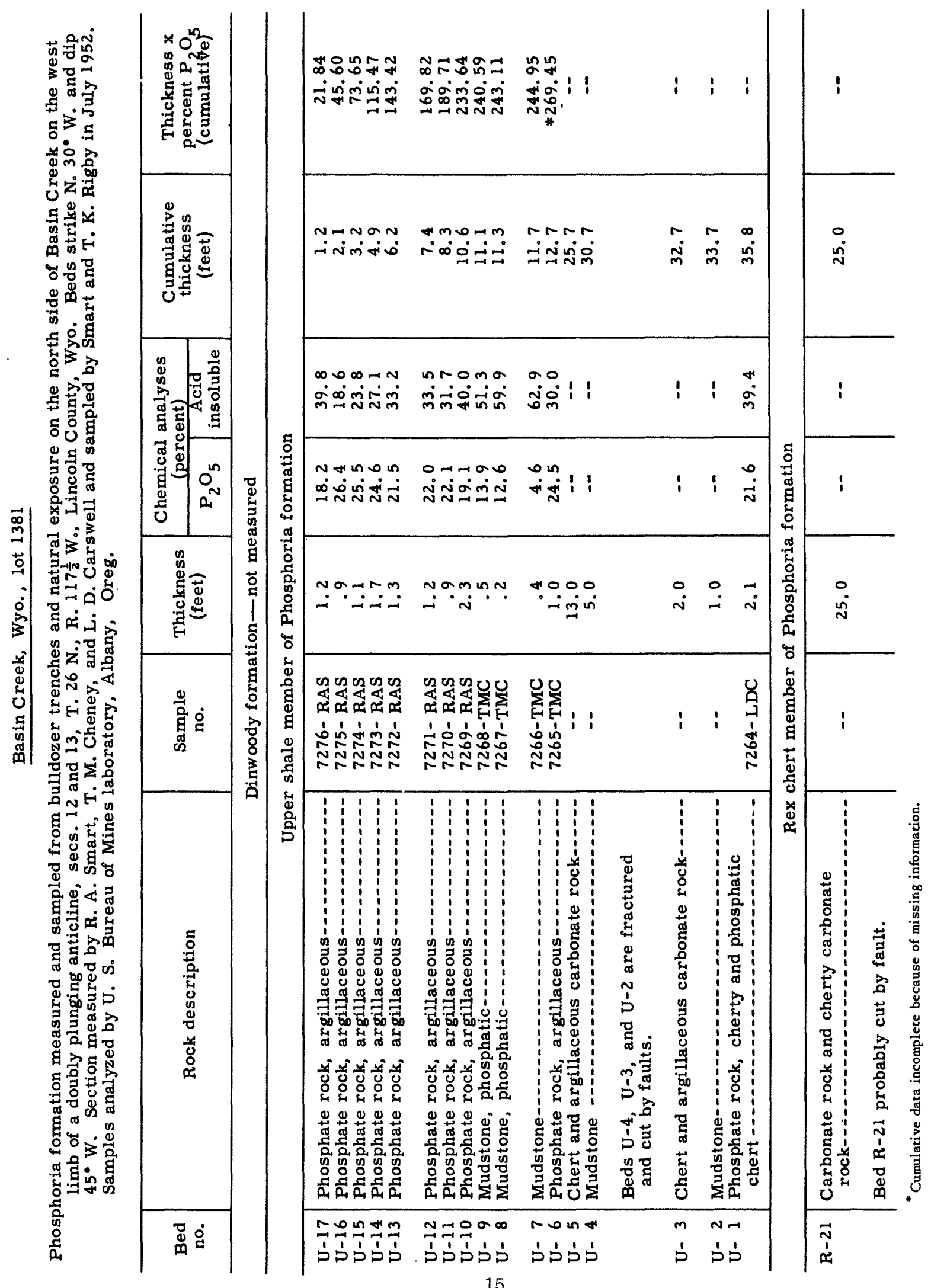




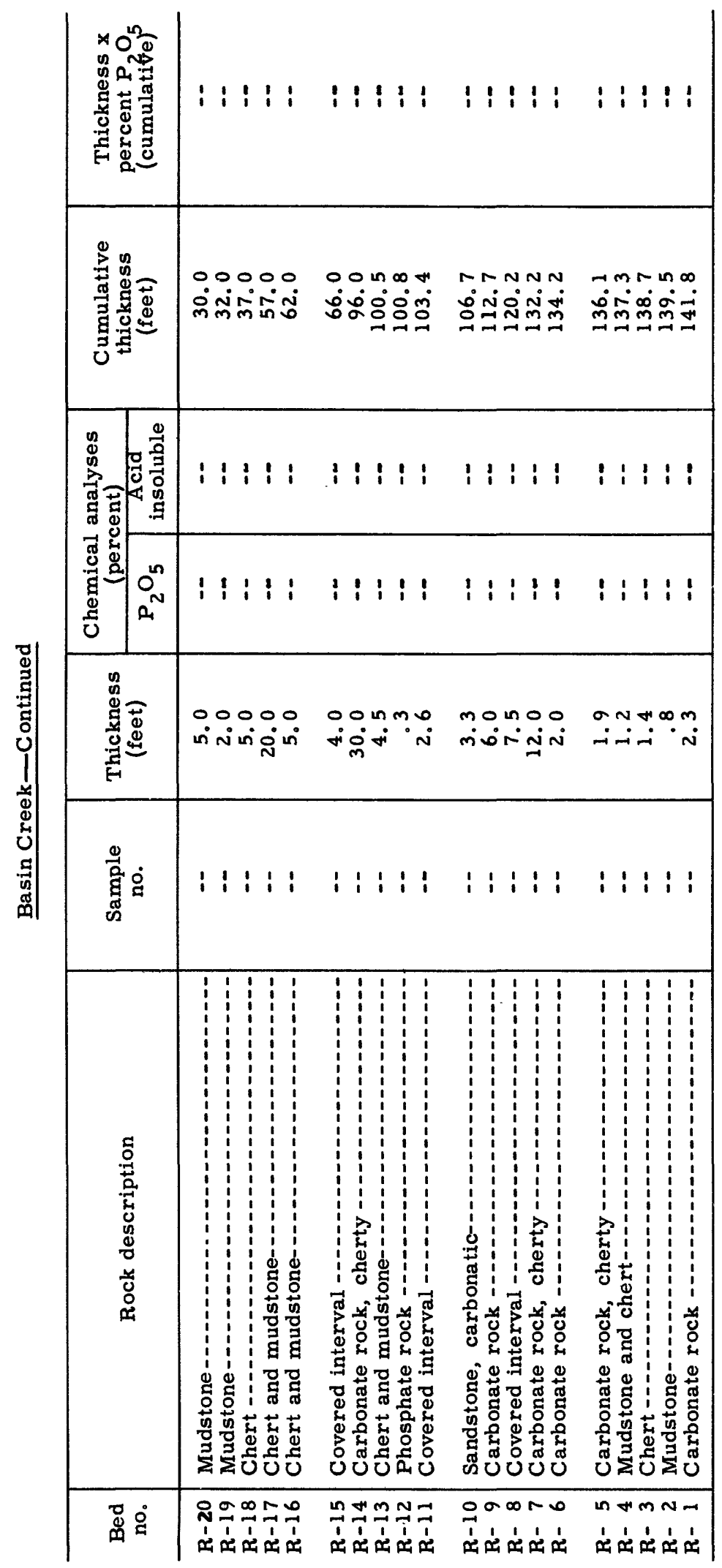

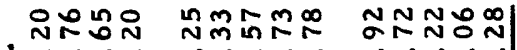

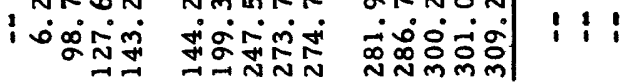

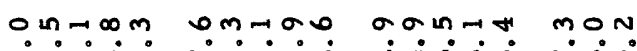

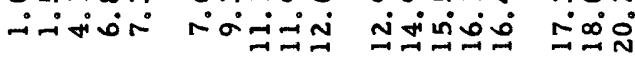

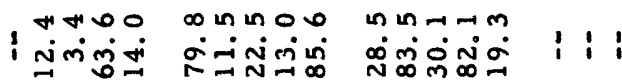

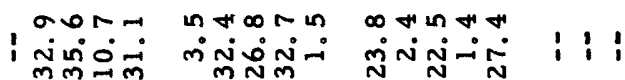

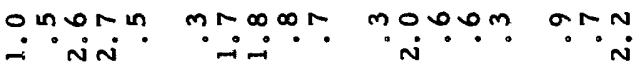

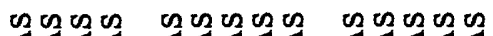

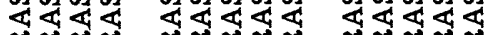

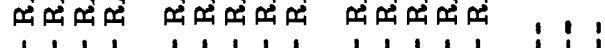

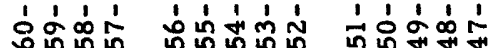

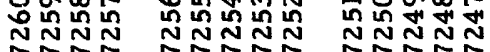

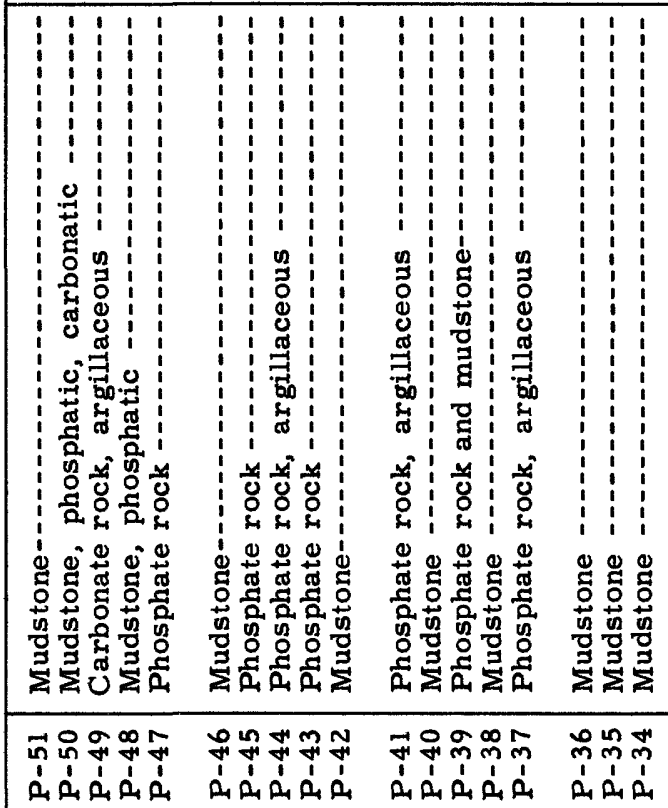




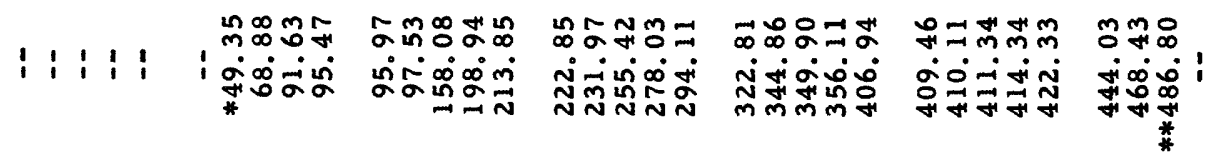

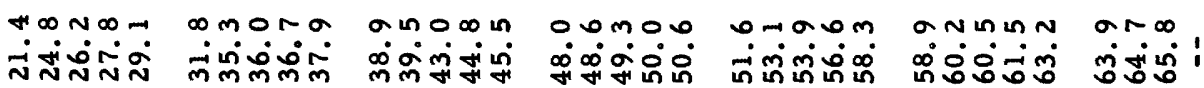

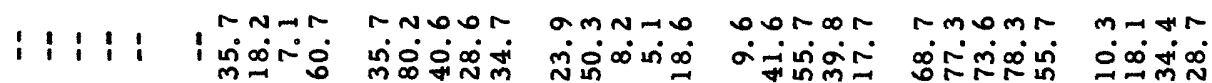

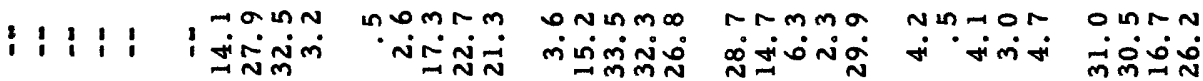

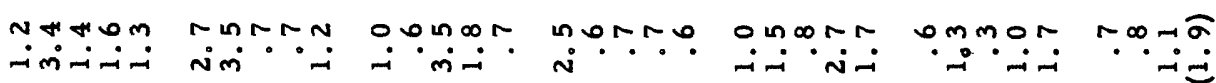

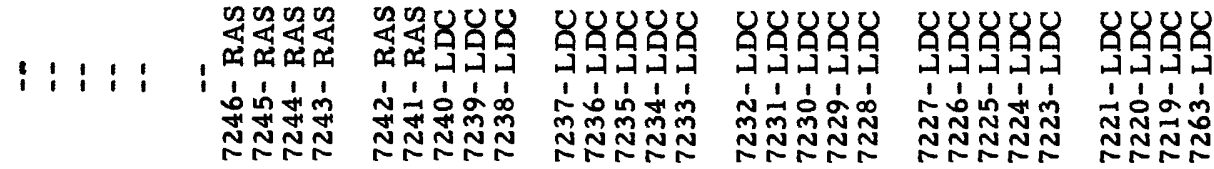

\begin{tabular}{|c|c|c|c|c|c|c|c|c|}
\hline 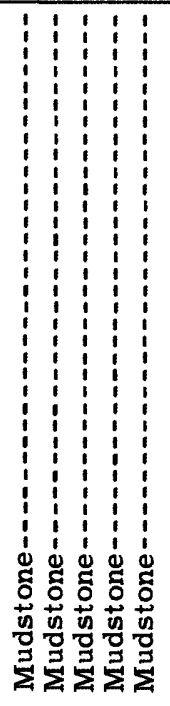 & 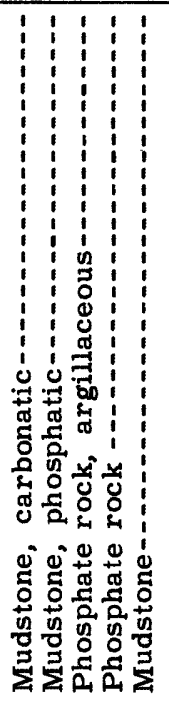 & 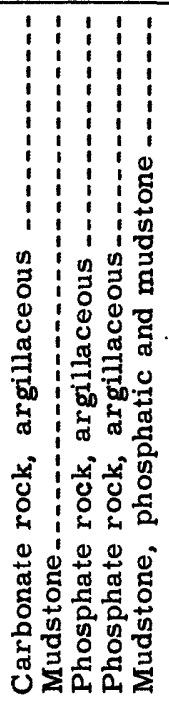 & 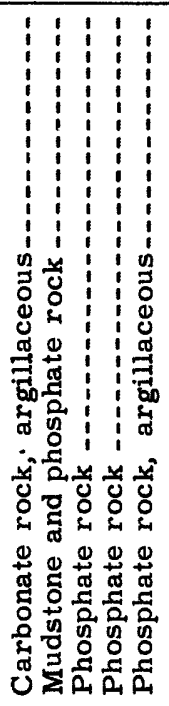 & 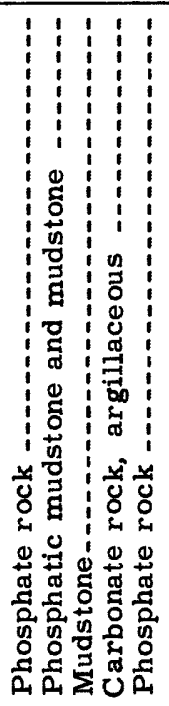 & 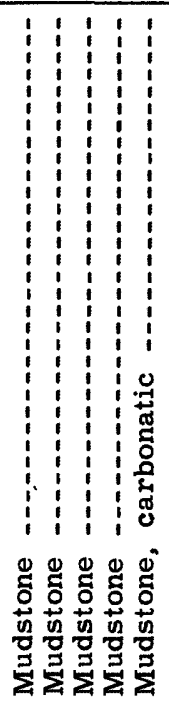 & 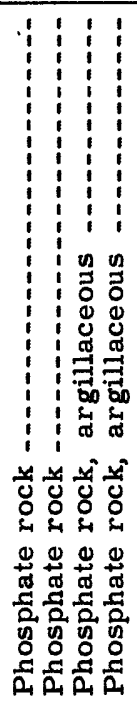 & 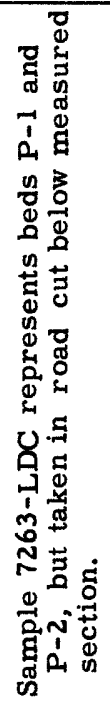 & 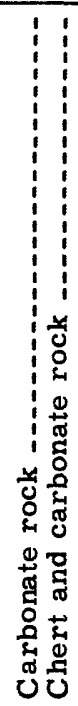 \\
\hline 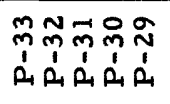 & 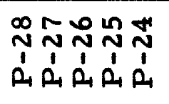 & 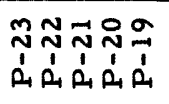 & 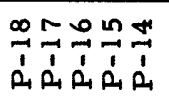 & 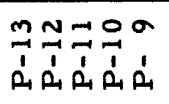 & 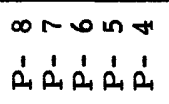 & $\begin{array}{l}m N-{ }^{\prime} \\
\dot{m}^{\prime} \dot{\alpha}^{\prime} \dot{\alpha}^{\prime}\end{array}$ & & $\begin{array}{l}T \pi \\
3 \\
\vdots \\
U \\
U\end{array}$ \\
\hline
\end{tabular}




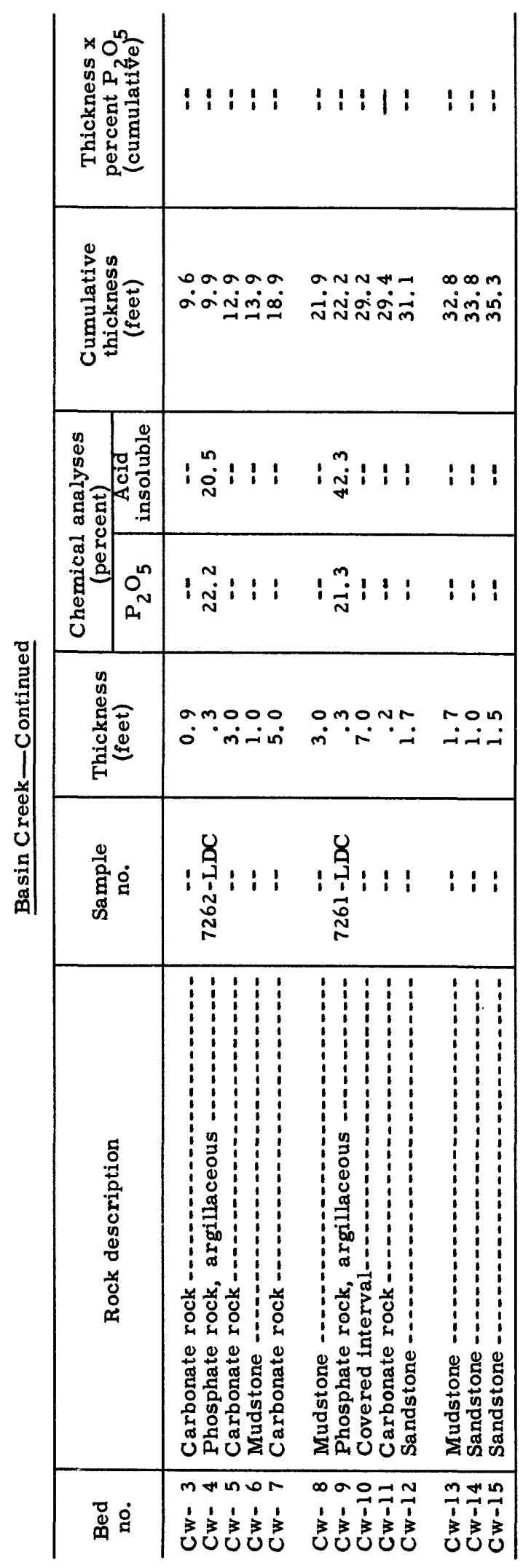




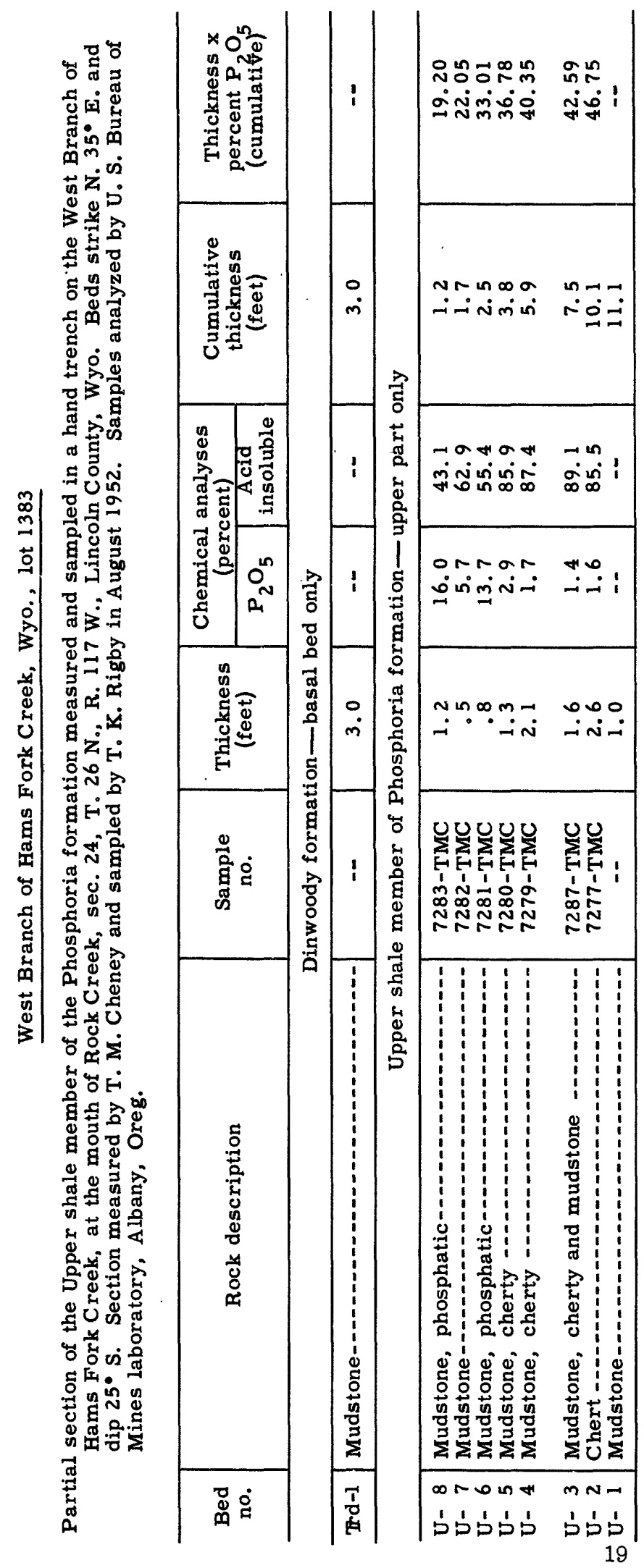




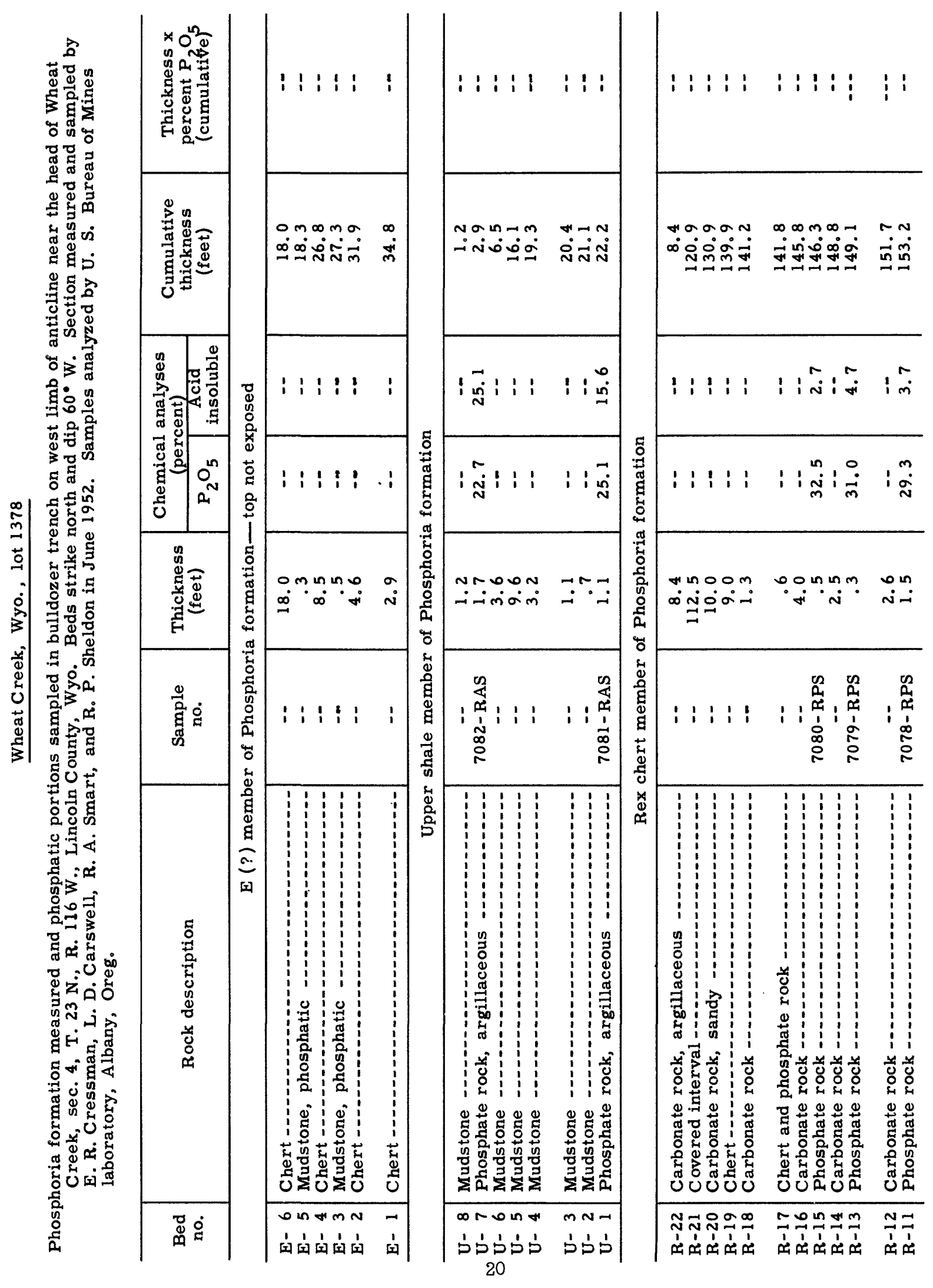




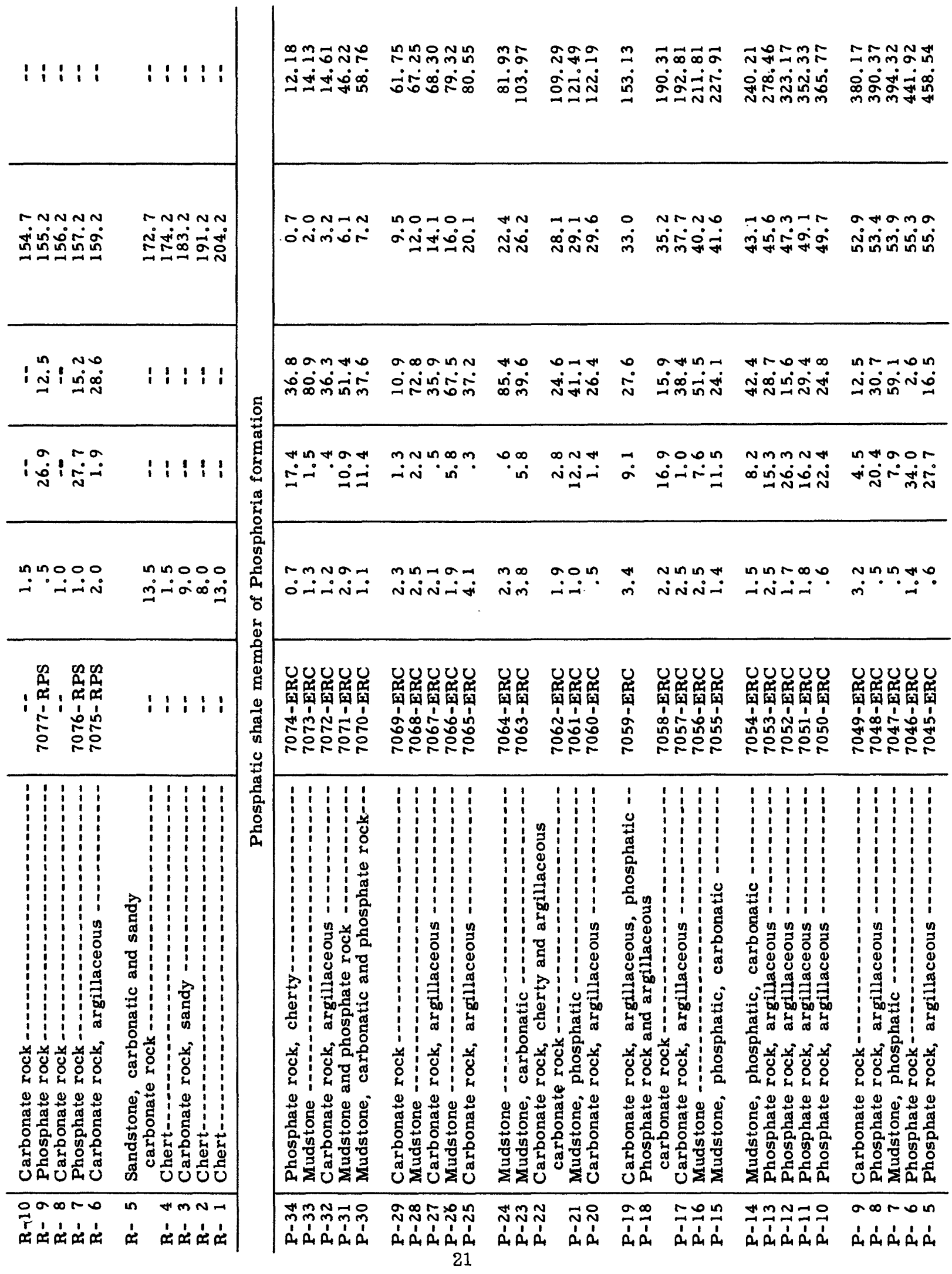




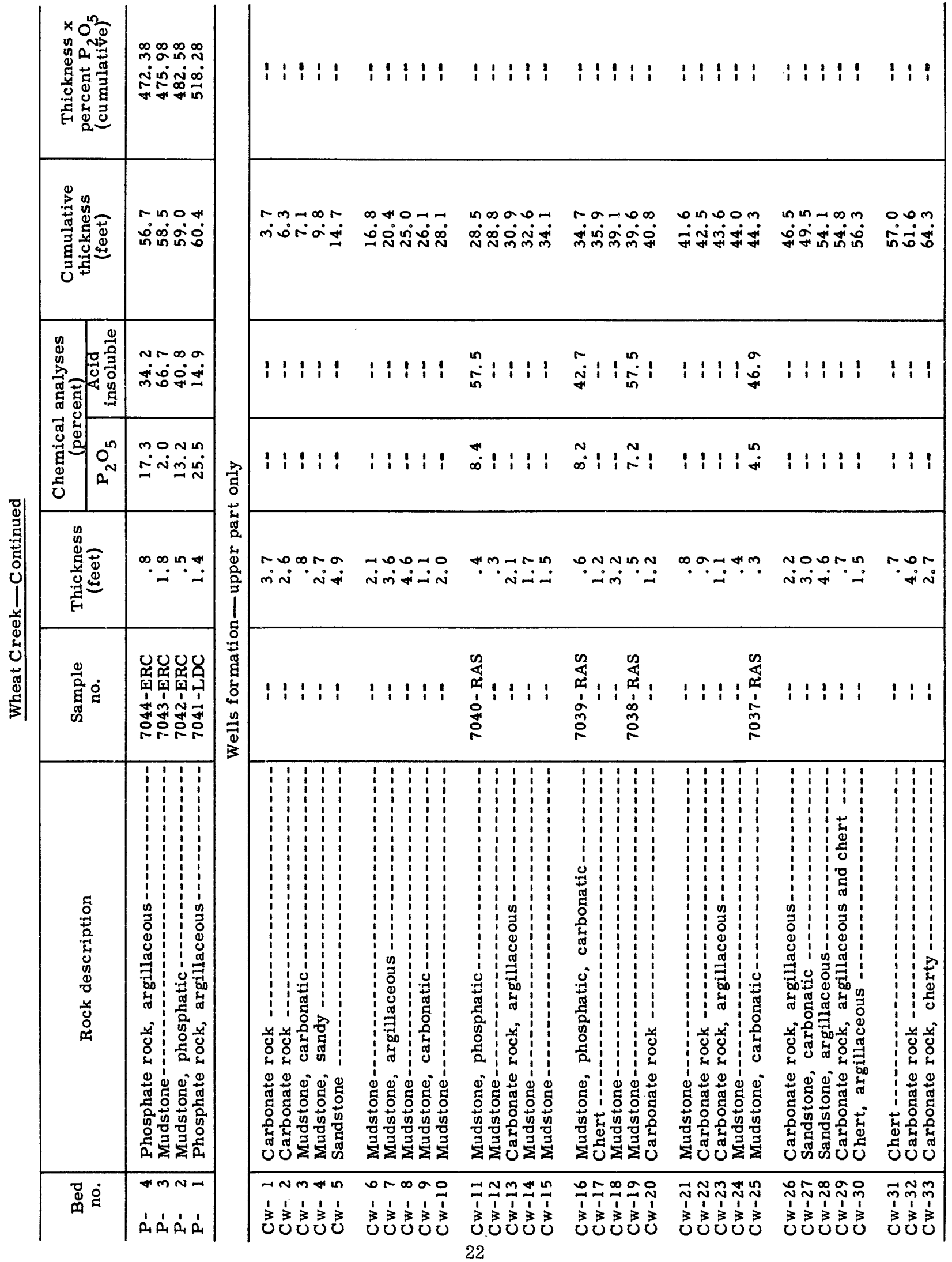




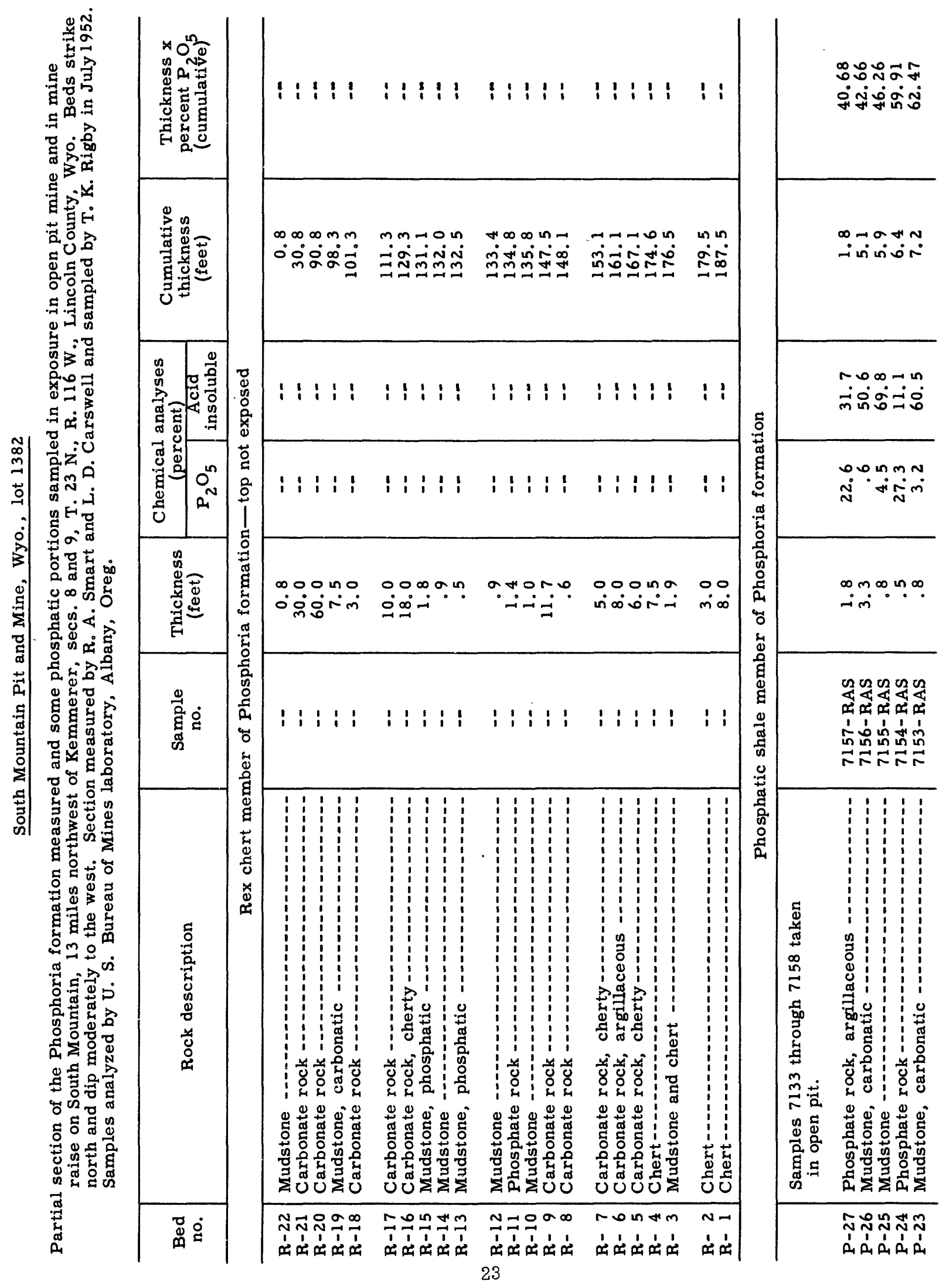




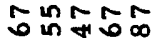

๓ूंडี่ง

어음
กดลูป

ลำํำ

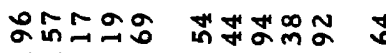

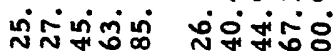

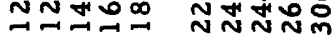

nin $\rightarrow a n$

aining

$000 N N$

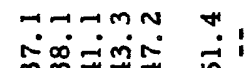

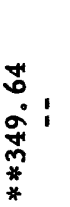

NतNलm

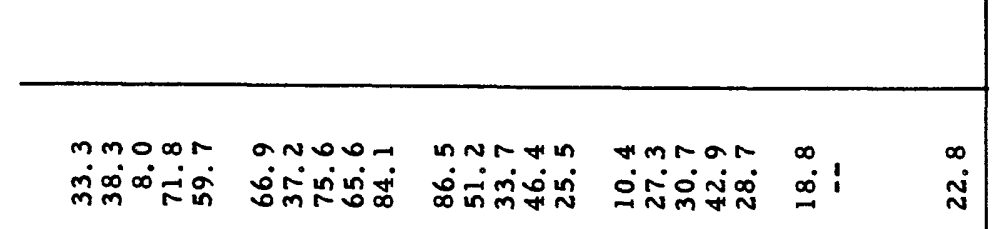

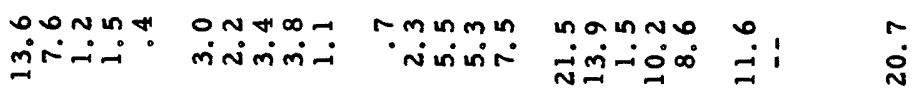

U⿱

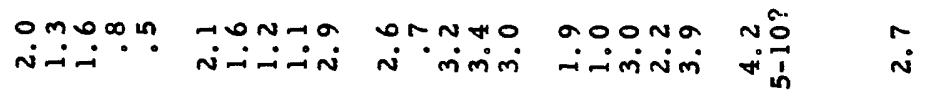

\begin{tabular}{|c|c|c|c|c|}
\hline 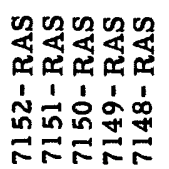 & 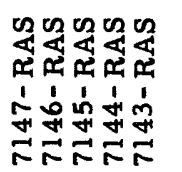 & 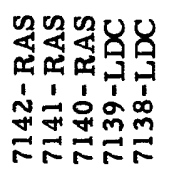 & 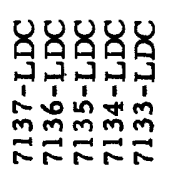 & 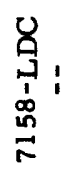 \\
\hline
\end{tabular}

\begin{tabular}{|c|c|c|c|c|c|c|}
\hline 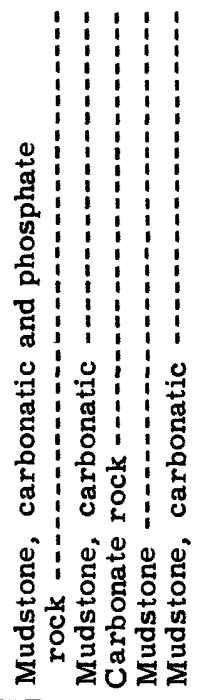 & 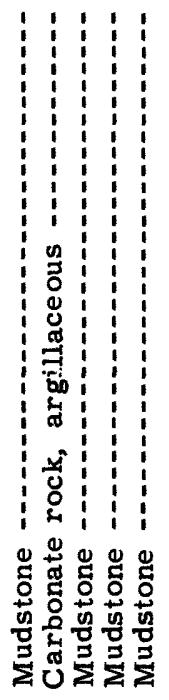 & 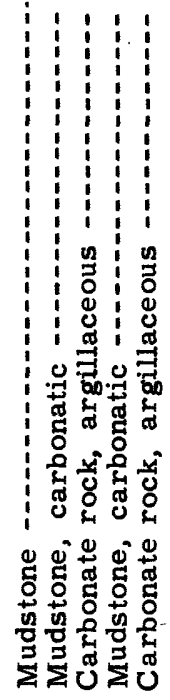 & 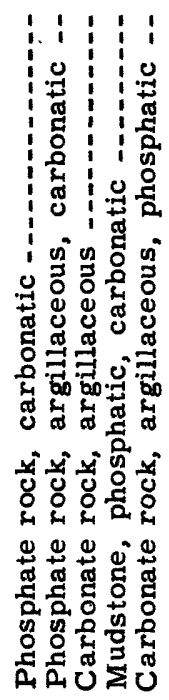 & 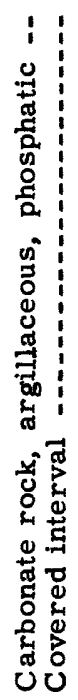 & 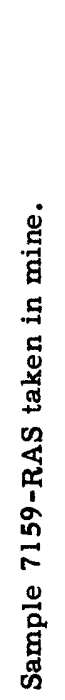 & 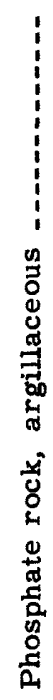 \\
\hline 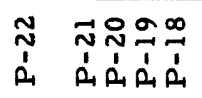 & 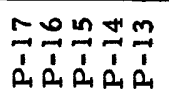 & 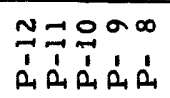 & 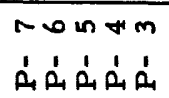 & n & & $\overrightarrow{\dot{n}}$ \\
\hline
\end{tabular}

